\title{
Percepção da qualidade de vida de idosos com ferida crônica
}

\author{
Perceptions on the quality of life of elderly people with chronic wound
}

Percepción de la calidad de vida de los ancianos con heridas crónicas

Recebido: 06/10/2019

Aprovado: 07/04/2020

Publicado: 01/07/2020

\section{Elayne Gonçalves Rodrigues do Nascimento ${ }^{1}$ Giovanna Gabrielly Custódio Macêdo ${ }^{2}$ Arthur Alexandrino ${ }^{3}$ Karla Karolline Barreto Cardins ${ }^{4}$ Fernanda Teixeira de Souza ${ }^{5}$ Matheus Figueiredo Nogueira ${ }^{6}$}

O objetivo deste estudo foi avaliar a percepção da qualidade de vida de idosos com feridas crônicas. Trata-se de estudo exploratório-observacional de natureza quantiqualitativa realizado com 20 idosos do Curimataú Paraibano, entre novembro de 2014 a março de 2015, através de um questionário sociodemográfico, do instrumento WHOQOL-Old e um roteiro de entrevista semiestruturado, analisados por análise descritiva, cálculos de escores e análise de conteúdo, respectivamente. Os resultados demonstraram que a qualidade de vida dos idosos encontra-se regular, com exceção das facetas "Participação social" e "Intimidade". Os depoimentos obtidos resultaram em três categorias: Percepção subjetiva da qualidade de vida; $O$ impacto físico e emocional da ferida crônica na qualidade de vida; e $O$ comprometimento da autonomia e das relações sociais em idosos com ferida crônica. Os discursos dos participantes enfatizaram os impactos físicos e emocionais da ferida crônica na sua qualidade de vida, sobretudo no comprometimento da autonomia e das relações sociais. Verifica-se a necessidade de implementação de intervenções de enfrentamento e a construção de linhas de cuidado específicas e interprofissionais, com foco na qualificação da assistência.

Descritores: Idoso; Saúde do idoso; Ferimentos e lesões; Qualidade de vida; Assistência integral à saúde.

This study aims to evaluate the perceptions on the quality of life of elderly people with chronic wound. This is an exploratory-observational study of quantitative and qualitative nature, carried out with 20 elderly people from the region of Curimataú, on the state of Paraíba, Brazil, between November of 2014 and March of 2015, through a sociodemographic questionnaire, the WHOQOL-Old tool and a semi-structured interview script, which we analyzed, respectively, by descriptive analysis, score calculations and content analysis. The results showed that the quality of life of elderly people is average, with the exception of the aspects "social participation" and "intimacy". The testimonies we gathered created three categories: Subjetive perception of quality of life; Physical and emotional impact of chronic wound on quality of life; and The impairment of autonomy and social relationships in elderly people with chronic wound. The participant's speeches emphasized the physical and emotional impacts of the chronic wound in their quality of life, especially in the impairment of autonomy and social relationships. There is a need to implement coping interventions and and build specific and interprofessional lines of care, with focus on the qualification of assistance.

Descriptors: Aged; Health of the elderly; Wounds and injuries; Quality of life; Comprehensive health care.

El objetivo de este estudio fue evaluar la percepción de la calidad de vida de los ancianos con heridas crónicas. Se trata de un estudio exploratorio, observacional de carácter cuantitativo y cualitativo realizado con 20 ancianos del territorio de Curimataú en la Paraíba, Brasil, entre noviembre de 2014 y marzo de 2015, mediante un cuestionario sociodemográfico, el instrumento WHOQOL-Old y un guion de entrevista semiestructurado, analizados mediante un análisis descriptivo, cálculos de puntuación y análisis de contenido, respectivamente. Los resultados mostraron que la calidad de vida de los ancianos es regular, con la excepción de las facetas "Participación social" e "Intimidad". Los testimonios obtenidos dieron lugar a tres categorías: Percepción subjetiva de la calidad de vida; El impacto físico y emocional de la herida crónica en la calidad de vida; y El deterioro de la autonomía y las relaciones sociales en ancianos con herida crónica. En los discursos de los participantes se destacaron los efectos físicos y emocionales de la herida crónica en su calidad de vida, especialmente el perjuicio de la autonomía y las relaciones sociales. Se verifica la necesidad de implementación de intervenciones de confrontación y la construcción de líneas de atención específicas e interprofesionales, con foco en la calificación de la asistencia.

Descriptores: Anciano; Salud del anciano; Heridas y traumatismos; Calidad de vida; Atención integral de salud.

1. Enfermeira. Especialista em Saúde da Família. Coordenadora de Atenção Básica de Remígio, PB, Brasil. ORCID: 0000-0001-6698-8830 E-mail: elaynegrnascimento@gmail.com

2. Enfermeira. Especializanda em Unidade de Terapia Intensiva (UTI) na modalidade Residência Uniprofissional pelo Hospital da Restauração da Universidade de Pernambuco, Recife, PE, Brasil. ORCID: 0000-0002-2365-0714 E-mail: cmacedogiovanna@hotmail.com

3. Graduando em Enfermagem da Universidade Federal de Campina Grande (UFCG), Campus Cuité, PB, Brasil. ORCID: 0000-0001-5817-4335 E-mail: alexandrinoarthurdm@gmail.com

4. Enfermeira. Mestre em Saúde Pública. Cuité, PB, Brasil. ORCID: 0000-0002-5571-2932 E-mail: karla_karolline@hotmail.com

5. Enfermeira. Especializanda em UTI, Urgência e Emergência. Especialista em Pesquisa em Saúde, João Pessoa, PB, Brasil. ORCID: 0000-0001-9193-5992 E-mail: fefeteixeira@outlook.com

6. Enfermeiro. Especialista em Saúde da Família. Mestre Enfermagem na Atenção à Saúde. Doutor em Saúde Coletiva. Professor Adjunto do Curso de Graduação em Enfermagem da UFCG, Cuité/PB, Brasil. ORCID: 0000-0002-5787-7861 E-mail: matheusnogueira.ufcg@gmail.com 


\section{INTRODUÇÃO}

A mudança no perfil demográfico e epidemiológico brasileiro caracteriza-se A prioritariamente pelo envelhecimento da população e alteração do padrão de 1 morbimortalidade, com aumento da prevalência das doenças e agravos crônicos ${ }^{1}$. Dentre as condições crônicas que apresentam elevados índices de prevalência e incidência, assim como demasiados impactos financeiros e sociais, destacam-se as feridas crônicas, ou seja, soluções de continuidade que não evoluem funcional e anatomicamente em um período de três meses, estando em geral associadas a lesões por pressão e a úlceras diabéticas e vasculogênicas crônicas, comuns na população idosa².

Essa parcela da população constitui um dos grupos de maior vulnerabilidade para o desenvolvimento de feridas crônicas em virtude das alterações celulares, tegumentares, vasculares e sistêmicas habitualmente associadas ao processo de envelhecimento e à coexistência de agravos, como insuficiência venosa e arterial, hipertensão arterial sistêmica e diabetes mellitus ${ }^{2,3}$.

As feridas crônicas determinam prejuízos globais na qualidade de vida do indivíduo acometido. Os impactos englobam mudanças complexas nos aspectos sociais, financeiros, físicos e psicológicos da vida diária, que contribuem para situações como isolamento social, maior grau de dependência, menor produtividade e baixa satisfação pessoal ${ }^{4}$.

Em virtude desses impactos e por abranger um conceito multidimensional relacionado à percepção do indivíduo sobre sua condição/situação, a qualidade de vida torna-se um parâmetro relevante para avaliação em condições crônicas, de maneira a viabilizar a construção de um cuidado multidisciplinar, resolutivo, humanizado e integral ${ }^{5}$. Nessa perspectiva, o objetivo deste estudo foi avaliar a percepção da qualidade de vida de idosos com feridas crônicas.

\section{MÉTODO}

Trata-se de um estudo de campo do tipo exploratório-observacional de natureza quantiqualitativa. 0 estudo foi realizado nas cidades de Barra de Santa Rosa, Cuité, Nova Floresta, Remígio e Sossego, componentes da microrregião do Curimataú Ocidental paraibano, norteado a partir do projeto de pesquisa maior intitulada: "Qualidade de vida de idosos e a diversidade de vivenciar o envelhecimento: um estudo no Curimataú Ocidental paraibano".

0 projeto maior se baseou em amostra probabilística do tipo aleatória simples, calculada com base em uma prevalência estimada de qualidade de vida satisfatória de $50 \%$ e considerando o erro amostral de 5\%, nível de confiabilidade de $95 \%$ e acréscimo de $20 \%$ para possíveis perdas. Conforme dados censitários do Instituto Brasileiro de Geografia e Estatística ${ }^{6}$, publicados em 2010, os cinco municípios possuíam um total de 9.606 habitantes com idade igual ou superior a 60 anos distribuídos da seguinte forma: 2.001 em Barra de Santa Rosa, 3.040 em Cuité, 1.576 em Nova Floresta, 2.614 em Remígio e 375 em Sossego, público que baseou o cálculo de amostragem. Baseando-se na premissa da representatividade, aplicou-se o cálculo de amostra probabilística sistemática.

Os participantes foram incluídos na pesquisa a partir do cumprimento dos seguintes critérios: I) ter idade igual ou superior a 60 anos; II) apresentar quadro de feridas crônica (para participação específica da entrevista); III) ser devidamente cadastrado e acompanhado na Estratégia de Saúde da Família; e IV) não possuir deficiência cognitiva, avaliada por indicadores do Mini-Exame do Estado Mental (MEEM).

A operacionalização da coleta de dados foi mediada pelos instrumentos: a) Questionário socioeconômico e demográfico, validado no Projeto "Situação de Saúde, Qualidade de Vida e Representações Sociais" do Grupo Internacional de Estudos e Pesquisas sobre Envelhecimento e Representações Sociais do Programa de Pós-Graduação em Enfermagem da Universidade Federal da Paraíba, vinculado ao Ministério da Saúde (do Brasil), para colher informações 
pessoais e o perfil social dos participantes; b) Questionário WHOQOL-Old, instrumento de mensuração e avaliação da qualidade de vida elaborado pela Organização Mundial de Saúde e validado no Brasil em 20067, organizado em seis facetas: "Funcionamento Sensório" (FS), "Autonomia" (AUT), "Atividades Passadas, Presentes e Futuras" (PPF), "Participação Social" (PSO), "Morte e Morrer" (MEM) e "Intimidade" (INT); e, c) roteiro de entrevista, solidificando o segmento qualitativo dos questionamentos: 1) 0 que significa Qualidade de Vida para você? 2) o que mais interfere na sua Qualidade de Vida como um portador de ferida crônica? 3) Como você avalia a sua Qualidade de Vida com a ferida crônica? enquanto itens sobre a percepção do idoso acometido por ferida crônica acerca da sua qualidade de vida.

A coleta de dados foi realizada durante os meses de novembro de 2014 a março de 2015. Após a identificação das Estratégias de Saúde da Família de adscrição da amostra específica da pesquisa, os enfermeiros e agentes comunitários de saúde foram contatados para fornecerem informações acerca da acessibilidade dos pesquisadores aos idosos.

Uma vez que o idoso aceitou participar do estudo e se encontrava de acordo com os critérios de inclusão, a equipe de pesquisadores procediam à coleta das informações, seja no domicílio ou na Unidade de Saúde da Família. Anteriormente ao preenchimento dos instrumentos, os pesquisadores apresentaram o Termo de Consentimento Livre e Esclarecido e informações gerais sobre a pesquisa, sendo este assinado em duas vias. A equipe de pesquisadores foi composta pelo pesquisador responsável, cinco enfermeiros egressos do Centro de Educação e Saúde da Universidade Federal de Campina Grande e 13 alunos regularmente matriculados no curso de Bacharelado em Enfermagem da instituição.

Os dados de caráter quantitativo, originados do Questionário de Coleta de Dados e cálculos dos escores relativos à avaliação da qualidade de vida adotada pelo WHOQOL-Old, foram digitados no programa Excel versão 2010, processados pelo software IBM Statistical Package for the Social Sciences versão 20 e calculados sob frequências simples, para as variáveis nominais e ordinais, e medidas de posição e variabilidade para as variáveis quantitativas. Para os cálculos do WHOQOL-Old, usou-se o somatório das respostas apontadas pelos idosos participantes do estudo para cada questão, dividido pelo número de respondentes ${ }^{8}$. As médias foram descritas (resultado da variante entre 1 e 5) referindo-se ao Escore Padronizado (EP). De modo genérico, escores elevados representam uma qualidade de vida satisfatória e escores baixos representavam uma qualidade de vida pouco satisfatória.

Os dados qualitativos, por sua vez, foram submetidos à Análise de Conteúdo $^{9}$ na modalidade de categoria temática, dando subsídio ao agrupamento dos depoimentos e a respectiva discussão.

A realização da pesquisa se deu conforme autorização do Centro de Educação e Saúde da Universidade Federal de Campina Grande e das cinco Secretarias Municipais de Saúde dos municípios envolvidos. 0 projeto foi submetido à apreciação do Comitê de Ética em Pesquisa do Hospital Universitário Alcides Carneiro e aprovado sob parecer no 844.702 e Certificado de Apresentação para Apreciação Ética 34715614.5.0000.5182.

\section{RESULTADOS}

Participaram do estudo 444 idosos, sendo 92 de Barra de Santa Rosa, 140 de Cuité, 73 de Nova Floresta, 122 de Remígio e 17 de Sossego. Dentre os 444 participantes, 20 idosos referiram a presença de ferida crônica, considerado neste trabalho.

A caracterização socioeconômica e demográfica está apresentada na Tabela 1, exibindo as seguintes variáveis: faixa etária, sexo, estado civil, arranjo familiar, presença de cuidador, identificação do cuidador, religião, alfabetização funcional, escolaridade e renda familiar.

Observou-se que $60 \%$ da amostra era do sexo feminino e com faixa etária média de 74,25 anos, variando de 60 a 93 anos. A maior parte dos idosos era casada (60\%), com arranjo familiar trigeracional (35\%), vivam somente com o cônjuge (20\%) ou com o cônjuge e filhos (20\%). 
Sobre a presença de cuidador, 55\% dos idosos afirmaram possuir cuidador domiciliar que, em sua maioria, eram os filhos (30\%).

Em grande expressividade religiosa, $85 \%$ dos idosos pertenciam à religião Católica Apostólica Romana. No que diz respeito ao alfabetismo funcional e grau de escolaridade, 55\% dos idosos eram analfabetos funcionais e apresentavam uma média de 2,05 anos de estudo. A média da renda familiar mensal se concentrou em $\mathrm{R} \$ 1.377,35$, oscilando entre a mínima de $\mathrm{R} \$$ 788,00 e a máxima de $\mathrm{R} \$ 1.576,00$ (Tabela 1 ).

Tabela 1. Perfil socioeconômico e demográfico simplificado de idosos com ferida crônica acompanhados pela Estratégia Saúde da Família do Curimataú Ocidental Paraibano (n=20). Paraíba, Brasil, 2015.

\begin{tabular}{|c|c|c|c|}
\hline \multirow{2}{*}{ Variável } & \multirow{2}{*}{ Categorias } & \multicolumn{2}{|c|}{ Idosos pesquisados } \\
\hline & & $\mathrm{f}$ & $\%$ \\
\hline \multirow[t]{3}{*}{ Faixa etária } & 60 a 74 & 11 & 55,0 \\
\hline & 75 a 89 & 07 & 35,0 \\
\hline & Acima de 90 & 02 & 10,0 \\
\hline \multirow[t]{2}{*}{ Medidas Idade } & Média $=74,25$ & & \\
\hline & Desvio padrão = 11,187 & Mínima = 60 & Máxima = 93 \\
\hline \multirow[t]{2}{*}{ Sexo } & Feminino & 12 & 60,0 \\
\hline & Masculino & 08 & 40,0 \\
\hline \multirow[t]{3}{*}{ Estado civil } & Solteiro & 01 & 5,0 \\
\hline & Casado & 12 & 60,0 \\
\hline & Viúvo & 07 & 35,0 \\
\hline \multirow[t]{6}{*}{ Arranjo familiar } & Sozinho & 03 & 15,0 \\
\hline & Somente com o cônjuge & 04 & 20,0 \\
\hline & Cônjuge e filhos & 04 & 20,0 \\
\hline & Cônjuge, filhos, genro ou nora & 01 & 5,0 \\
\hline & Somente com os filhos & 01 & 5,0 \\
\hline & Arranjos trigeracionais & 07 & 35,0 \\
\hline \multirow[t]{2}{*}{ Presença de cuidador } & Sim & 11 & 55,0 \\
\hline & Não & 09 & 45,0 \\
\hline \multirow[t]{5}{*}{ Identificação do cuidador } & Cônjuge & 01 & 5,0 \\
\hline & Somente os filhos & 06 & 30,0 \\
\hline & Outro idoso & 01 & 5,0 \\
\hline & Cuidador particular & 03 & 15,0 \\
\hline & Não tem cuidador & 09 & 45,0 \\
\hline \multirow[t]{2}{*}{ Religião } & Católica & 17 & 85,0 \\
\hline & Evangélica & 03 & 15,0 \\
\hline \multirow[t]{2}{*}{ Alfabetização funcional } & Sim & 09 & 45,0 \\
\hline & Não & 11 & 55,0 \\
\hline \multirow[t]{3}{*}{ Escolaridade } & Não alfabetizado & 11 & 55,0 \\
\hline & 01 a 04 anos estudados & 04 & 20,0 \\
\hline & 05 a 08 anos estudados & 05 & 25,0 \\
\hline \multirow[t]{2}{*}{ Medidas escolaridade } & Média = 2,05 & & \\
\hline & Desvio padrão $=2,481$ & Mínima = 0 & Máxima = 6 \\
\hline \multirow[t]{2}{*}{ Renda Familiar } & Média $=1.377,35$ & & \\
\hline & Desvio padrão $=271,546$ & Mínima = 788,00 & Máxima $=1.576,00$ \\
\hline Total & & 20 & 100,0 \\
\hline
\end{tabular}

Conforme as respostas dos participantes e as facetas "Funcionamento Sensório", "Autonomia", "Atividades Passadas, Presentes e Futuras", "Participação Social", "Morte e Morrer" e "Intimidade" que compõem o questionário WHOQOL-Old, a Tabela 2 expõe o cálculo das medidas descritivas dos itens de cada faceta.

Dentre os 24 itens contidos no WHOQOL-Old, o melhor EP referiu-se ao item 15 da faceta "Atividades Passadas, Presentes e Futuras", que trata da avaliação da satisfação dos idosos participantes do estudo com o que alcançaram na vida $(m=4,00)$. Em contrapartida, o pior 
escore foi identificado no item 04 da faceta "Autonomia", que diz respeito à intensidade com que os idosos controlam o próprio futuro $(\mathrm{m}=1,95)$.

Tabela 2. Medidas descritivas dos itens do WHOQOL-Old* em idosos com feridas crônicas acompanhados na Estratégia Saúde da Família do Curimataú Ocidental Paraibano $(\mathrm{n}=20)$. Paraíba, Brasil, 2015.

\begin{tabular}{|c|c|c|c|}
\hline Faceta/Questão & m (EP) & dp & cv (\%) \\
\hline \multicolumn{4}{|l|}{ Funcionamento Sensório (FS) } \\
\hline Old_01 - Intensidade da perda dos sentidos na vida diária ** & 2,90 & 1,21 & 41,71 \\
\hline Old_02 - Intensidade da perda dos sentidos na participação em atividades ** & 3,05 & 1,19 & 39,05 \\
\hline Old_10 - Efeito de funcionamento dos sentidos na capacidade de interação ** & 2,95 & 1,10 & 37,26 \\
\hline Old_20 - Avaliação do funcionamento dos sentidos & 2,80 & 0,95 & 33,98 \\
\hline \multicolumn{4}{|l|}{ Autonomia (AUT) } \\
\hline Old_03 - Liberdade para tomar as próprias decisões & 3,40 & 1,31 & 38,64 \\
\hline Old_04 - Intensidade em que controla o próprio futuro & 1,95 & 1,15 & 58,77 \\
\hline Old_05 - Intensidade em que as pessoas respeitam a sua liberdade & 3,80 & 0,89 & 23,54 \\
\hline Old_11 - Capacidade de fazer as coisas que gostaria & 2,30 & 1,26 & 54,81 \\
\hline \multicolumn{4}{|l|}{ Atividades Passadas, Presentes e Futuras (PPF) } \\
\hline Old_12 - Capacidade de satisfação com oportunidades para realizações na vida & 3,00 & 1,08 & 35,87 \\
\hline Old_13 - Capacidade de sentir que recebeu o merecido reconhecimento na vida & 3,80 & 0,77 & 20,20 \\
\hline Old_15 - Avaliação da satisfação com o que alcançou na vida & 4,00 & 0,92 & 22,94 \\
\hline Old_19 - Avaliação da felicidade em coisas a esperar no futuro & 3,05 & 0,89 & 29,08 \\
\hline \multicolumn{4}{|l|}{ Participação Social (PSO) } \\
\hline Old_14 - Capacidade de ter o suficiente para fazer em cada dia & 2,00 & 1,12 & 56,20 \\
\hline Old_16 - Avaliação da satisfação com a maneira com a qual usa o tempo & 2,80 & 1,20 & 42,73 \\
\hline Old_17 - Avaliação da satisfação com o nível de atividades & 2,20 & 0,95 & 43,25 \\
\hline Old_18 - Avaliação da satisfação em participar de atividades na comunidade & 2,20 & 1,06 & 48,01 \\
\hline \multicolumn{4}{|l|}{ Morte e morrer (MEM) } \\
\hline Old_06 - Intensidade da preocupação com a maneira pela qual irá morrer ** & 2,95 & 1,47 & 49,77 \\
\hline Old_07 - Intensidade do medo de não poder controlar a morte ** & 2,95 & 1,36 & 45,98 \\
\hline Old_08 - Intensidade do medo de morrer $* *$ & 3,15 & 1,60 & 50,75 \\
\hline Old_09 - Intensidade do temor em sofrer dor antes de morrer ** & 2,90 & 1,41 & 48,64 \\
\hline \multicolumn{4}{|l|}{ Intimidade (INT) } \\
\hline Old_21 - Intensidade do sentimento de companheirismo na vida & 2,80 & 1,79 & 64,10 \\
\hline Old_22 - Intensidade do sentimento de amor em sua vida & 2,70 & 1,78 & 65,93 \\
\hline Old_23 - Intensidade das oportunidades para amar & 2,10 & 1,59 & 75,53 \\
\hline Old_24 - Intensidade das oportunidades para ser amado & 2,05 & 1,50 & 73,34 \\
\hline
\end{tabular}

A compreensão geral do desempenho dos idosos referente a cada uma das facetas pode ser observada na Figura 1, a partir da análise do Escore Transformado das Facetas (ETF) que expressa a relação entre o valor mais baixo possível (0\%) e o mais alto possível (100\%), e do Escore Transformado Total (ETT), que demonstra a média percentual da qualidade de vida dos idosos considerando todas as facetas do questionário por meio da categorização a seguir: 0 $20 \%$ = qualidade de vida péssima; $20-40 \%$ = qualidade de vida ruim; $40-60 \%=$ qualidade de vida regular; $60-80 \%$ = qualidade de vida boa; $80-100 \%$ = qualidade de vida excelente.

A faceta "Atividades Passadas, Presentes e Futuras" apresentou o melhor desempenho, classificando a qualidade de vida nesse aspecto como boa (61,56\%). 0 pior desempenho foi obtido pela faceta "Participação Social" (32,50\%), determinando um significativo comprometimento da qualidade de vida neste aspecto. Por meio da conversão do ETF em ETT, a qualidade de vida geral dos idosos participantes desse estudo encontra-se regular (45,94\%).

Os depoimentos obtidos resultaram em três categorias: I) Percepção subjetiva da qualidade de vida; II) $O$ impacto físico e emocional da ferida crônica na qualidade de vida; e III) o comprometimento da autonomia e das relações sociais em idosos com ferida crônica. 


\section{Percepção subjetiva da qualidade de vida}

Os idosos relacionaram o significado de qualidade de vida a quatro fatores principais: saúde, suprimento das necessidades, presença da família e liberdade para sair de casa. A palavra saúde se apresentou como sinônimo de qualidade de vida na maioria dos discursos:

É a minha saúde [...] (G2)

Só saúde. (G4)

Saúde, união, principalmente a saúde. (G5)

[...] Cuidar da saúde e procurar ajuda logo quando tiver a ferida. (G7)

[...] a saúde, ela sim é boa. (G8)

[...] assim, é ter saúde, vida boa. (G10)

Funcionamento Sensório

Autonomia

Atividades Passadas, Presentes e Futuras

Participação Social

Morte e Morrer

Intimidade

Total

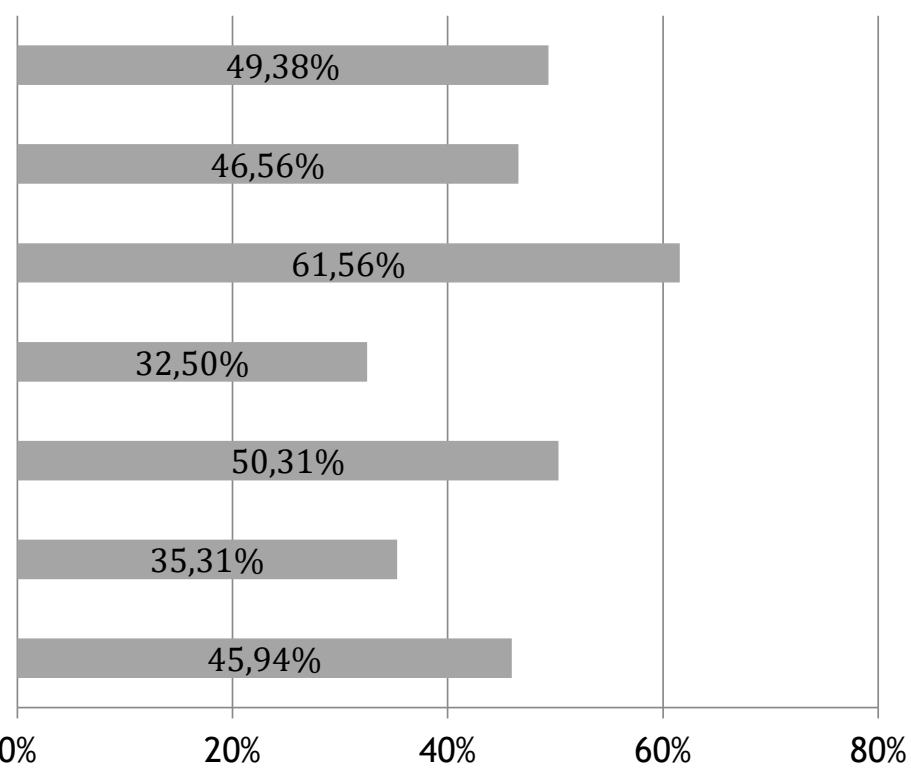

Figura 1. Escore Transformado das Facetas e Escore Transformado Total do questionário WHOQOL-Old em idosos com feridas crônicas acompanhados na Estratégia Saúde da Família do Curimataú Ocidental Paraibano. Paraíba, Brasil, 2015.

\section{O impacto físico e emocional da ferida crônica na qualidade de vida}

Os discursos destacaram:

Falta minha circulação [...] eu mesmo não posso fazer mais nada [...]. (G9)

Minhas veias atrapalham muito [...] e pra andar, é ruim, porque nem todo tempo eu 'tô' legal. [...] vivo em muita solidão [...] minha vida eu não gosto, fico agoniada [...] eu me irrito, me maldigo muito [...]. (G10)

Meus 'quartos' está muito desgastado [...]. (G2)

Agora pra mim não "tá bom" mais não "né" [...], não posso nem me virar por cima de uma perna e nem da outra [...]. A enfermeira mandava eu dormir de 'papo pra cima' pra melhorar a dormida. (G6).

Fiquei mais triste [...]. Não saio mais na rua. (G3)

Péssima, muito ruim [...]. Tem que ficar bom para poder ter uma qualidade de vida. (G7)

[...] felicidade, isso eu não tenho mais. (G5)

Me sinto inútil [...] vejo tudo errado em minha vida. (G2)

O comprometimento da autonomia e das relações sociais em idosos com ferida crônica

As interferências causadas no convívio social e nas atividades de vida diária se mostram nas falas:

Ter vontade de fazer as coisas e não poder [...]. (G1)

o pior de tudo é que agora eu não posso andar mais [...] não posso tomar conta da minha casa [...]. É só isso. (G5)

Ah, o que mais interferiu foi eu não poder mais trabalhar. (G7) 


\section{DISCUSSÃO}

A faixa etária com maior representatividade foi a de idosos jovens com idade de 60 a 74 anos (55\%), seguido por aqueles com idades de 75 a 89 anos (35\%) e acima de 90 anos (10\%). Esse resultado corrobora uma pesquisa realizada em Teresina, Piauí, que aponta a idade como um dos mais relevantes fatores envolvidos no desenvolvimento de feridas crônicas, sendo facilmente observáveis nos indivíduos após os 60 anos de idade ${ }^{10}$.

A maioria dos idosos participantes do estudo é do sexo feminino (60\%). Assim como nesse estudo, uma pesquisa realizada com idosos que apresentavam feridas crônicas revelou que $74,07 \%$ dos entrevistados eram do sexo feminino. Este cenário segue a tendência da feminilização da velhice, e é justificado pela maior expectativa de vida do grupo ${ }^{10}$.

Em relação ao estado civil, $60 \%$ dos participantes eram casados, apresentando semelhança com um estudo realizado no município de Uberaba, Minas Gerais, em que a maior porcentagem do idosos entrevistados se mostravam casados ou morando com companheiro, aspecto esse que favorece o enfrentamento do idoso diante da situação e o cuidado com a ferida. Em contrapartida, um somatório considerável não possui companheiro (40\%), fato que tende a dificultar a terapêutica e gerar complicações de esfera física, social e psíquica ao idoso, como isolamento social e declínio da autoestima11.

Quanto aos arranjos, os trigeracionais atingiram 35\%. Este tipo de arranjo é uma composição familiar contemporânea que nem sempre é favorável à acomodação, além de colaborar para que as demandas dos idosos sejam preteridas em benefício dos mais jovens ${ }^{12}$.

A presença de cuidador foi verificada em $55 \%$ dos casos, e contribui para um menor risco de agravos e de recidivas por falta de cuidados específicos com a ferida. No entanto, $45 \%$ mencionaram não possuir cuidador. A ausência de cuidador faz com que os indivíduos desenvolvam habilidades práticas e assumam seus próprios cuidados ${ }^{11}$. Contudo, o idoso que assume o autocuidado pode apresentar desgaste físico e emocional maior, principalmente devido à rotina exaustiva que demandam os cuidados com a ferida.

Dos idosos que afirmaram ter um cuidador, a maioria mencionou se tratar dos filhos (30\%). 0 cuidador familiar tem importância ímpar no cuidado às condições de saúde dos indivíduos, com destaque para os doentes crônicos. Os componentes da família idealizam as formas de cuidado de acordo com a compreensão que possuem da situação e esse processo pode conduzir a uma ação conjunta dentro do contexto familiar ${ }^{11}$.

De maneira similar ao estado da Paraíba (75,1\%) e ao Brasil $(64,6 \%)$, grande parte dos idosos participantes pertencem à religião Católica Apostólica Romana (85\%). Apesar da existência de poucos estudos que associem o impacto da religião na qualidade de vida e na saúde, entende-se que alguns dos enfrentamentos efetivos para as mudanças biopsicossociais comuns aos idosos são as crenças espirituais e as práticas religiosas ${ }^{13}$.

A maior parte dos idosos eram analfabetos, com média de 2,05 anos de estudo, mostrando um perfil de analfabetismo funcional por apresentarem menos de quatro anos de estudos. Assim como no presente estudo, uma pesquisa realizada no município de João Pessoa, Paraíba, apontou que a maior parte dos idosos considerados vulneráveis eram analfabetos. A escolaridade é um determinante socioeconômico e, contribui para a desigualdade social, dificulta o acesso à educação em saúde e, por consequente, prejudica a adesão de condutas saudáveis que tendem a melhorar a qualidade de vida.

Ademais, o baixo nível de escolaridade entre os idosos favorece o aumento da utilização dos serviços de saúde, já que essa parcela da população é a mais acometida por condições crônicas que poderiam ter sido prevenidas durante toda vida ${ }^{4,14}$. Idosos com menor escolaridade tendem a ter uma maior necessidade de cuidadores para realizar as atividades do cotidiano, podendo ainda interferir na compreensão e assimilação dos cuidados a saúde.

A renda média familiar de $\mathrm{R} \$ 1.377,35$ mostra-se insuficiente para atender as necessidades humanas básicas. Corroborando com os achados da pesquisa, um estudo realizado com idosos com feridas crônicas na cidade de Teresina, Piauí, apontou que a maioria 
dos participantes apresentavam renda familiar de 1 a 3 salários mínimos, ou seja, pessoas de baixa renda. Com a renda insuficiente, o idoso não se alimenta de forma adequada, uma vez que sua condição financeira não permite atender todas as suas necessidades básicas².

A presença de feridas crônicas é uma considerável fonte adicional de despesas, pelo tratamento farmacológico e materiais para curativos. Traz-se aqui um fator capaz de desestabilizar as finanças familiares e afetar a qualidade de vida dos indivíduos ${ }^{15}$.

Conforme o EP do WHOQOL-Old, a faceta que obteve a melhor pontuação foi "Atividades Passadas, Presentes e Futuras", que trata da avaliação da satisfação dos idosos participantes do estudo com o que alcançaram na vida $(m=4,00)$, influenciando positivamente a qualidade de vida. Em contrapartida, o pior escore foi identificado no item 04 da faceta "Autonomia", que diz respeito à intensidade em que os idosos controlam o próprio futuro $(m=1,95)$. A ausência ou o baixo controle sobre o próprio futuro indica uma baixa qualidade de vida.

O baixo escore para a intensidade em que os idosos controlam o próprio futuro é um indicador negativo à qualidade de vida, uma vez que a autonomia constitui-se condição fundamental para um envelhecimento prazeroso e significativo ${ }^{16}$. No entanto, um estudo que analisou a execução de atividades de vida diária por portadores de feridas crônicas apontou que $42,9 \%$ afirmaram incomodar-se por não conseguirem realizar tarefas simples, de modo a impactar negativamente sua autonomia ${ }^{17}$. Este resultado é, portanto, comparável ao escore de 46,56\% para a satisfação quanto à autonomia como dimensão da qualidade de vida.

0 resultado da faceta "Atividades Passadas, Presentes e Futuras" mostra que os idosos desta pesquisa estão satisfeitos com as conquistas que alcançaram ao longo da vida e com as perspectivas para o futuro. 0 percentual expressivo para essa faceta pode ratificar que a satisfação é um fenômeno complexo e de difícil mensuração referindo-se, portanto, a uma condição subjetiva. A satisfação com a vida é um julgamento cognitivo de alguns domínios específicos, além de ser constituída de um processo de juízo e avaliação geral da própria vida de acordo com critérios próprios ${ }^{18}$.

No cálculo do ETF, a faceta "Atividades Passadas, Presentes e Futuras" também mostrou o melhor resultado, enquanto a "Participação Social (PS)" teve escores significativamente abaixo das demais. 0 baixo desempenho na PS demonstra a insatisfação dos idosos quanto às atividades do cotidiano, especialmente na comunidade, assim como insatisfação com o nível de atividade diária e com a utilização do tempo.

Este cenário pode ser justificado pelas limitações que a ferida crônica traz ao portador, como perda de sua liberdade, restrição na vida social e até mesmo imposição do uso de determinadas peças do vestuário. Todos esses fatores produzem repercussões psicoemocionais que refletem na autoimagem e autoestima do indivíduo, de modo a impactar no seu modo de se relacionar com os outros e, consequentemente, na sua qualidade de vida ${ }^{19}$.

Os determinantes relacionados à saúde têm permeado as mais diversas conceituações do termo qualidade de vida na velhice. Nesse sentido, acredita-se que as pessoas desfrutam de melhor qualidade de vida quando permanecem sadias à medida que envelhecem ${ }^{20}$. Entretanto, a ocorrência da ferida crônica envolve um processo complexo e patológico que causa alterações biológicas, emocionais, físicas e sociais que impõe consideráveis limitações e necessidades peculiares à vida do indivíduo 21 .

Em decorrência do comprometimento físico, o desgaste emocional dos idosos é percebido quando questionados sobre a sua qualidade de vida após a ferida. É notório que o portador de ferida crônica apresenta frequente discurso relacionado à perda do sentido de viver e falta de perspectivas para o futuro e desesperança.

Além do impacto emocional, a perda da autonomia e o prejuízo às relações sociais representam as maiores preocupações. Estudos afirmam que o maior efeito negativo da ferida se reflete na redução das atividades dos indivíduos, anteriormente ativos, em relação ao seu trabalho e tarefas cotidianas ${ }^{22,23}$, cenário também observado nos discursos dos idosos desta 
pesquisa ao serem questionados sobre a interferência da ferida em sua qualidade de vida, fato que, de maneira geral, contribui para a dependência familiar e o isolamento social ${ }^{4}$.

Aspectos clínicos associados à ferida crônica, como dor ao deambular, dor em repouso e uso de medicamentos para dor, apresentam-se como variáveis que diminuem estatisticamente a qualidade de vida do idoso, sobretudo por limitar sua mobilidade, causar desregulação do sono e repouso e alterar o humor ${ }^{4,5}$.

Dessa maneira, compreender a qualidade de vida de um idoso com ferida crônica requer reflexões que englobam não apenas o seu sofrimento físico, mas também aspectos que envolvem sua situação psicossocial e o impacto dessa condição para o indivíduo e seus familiares, que na maioria as vezes não compreendem a dimensionalidade que abarca tal problemática.

\section{CONCLUSÃO}

0 estudo demonstrou que idosos com ferida crônica apresentam qualidade de vida regular em quase todos os domínios do WHOQOL-Old, com exceção das facetas "Participação social" e "Intimidade". Esse achado culmina na indispensabilidade de cuidados especializados com as feridas executados por profissionais habilitados e competentes, para assim favorecer a (re)integração do idoso ao seu meio socio-familiar.

Sob a perspectiva subjetiva das entrevistas, mesmo nas facetas "Participação social" e "Intimidade", os discursos demonstram percepção de qualidade de vida contrária, já que todos os idosos, no geral, a consideram ruim, sobretudo pelas dificuldades impostas pela ferida, como seu impacto negativo no estado físico e emocional, na autonomia e nas relações sociais.

Os resultados obtidos sugerem a necessidade de se investir em intervenções de enfrentamento e estímulo à participação social, assim como na formulação de linhas de cuidado específicas e interprofissionais que assegurem boas condições à saúde e qualidade de vida diante do processo de envelhecimento e na presença de feridas. Além disso, tal disparidade incide sobre a necessidade de inserir a percepção do indivíduo sobre o seu estado de saúde na avaliação de condições crônicas.

A limitação deste estudo envolveu o reduzido número de participantes em virtude do recorte populacional delimitado. No entanto, por relacionar grupos específicos (idosos e portadores de feridas crônicas), permite a representatividade do local e período.

\section{REFERENCIAS}

1. Duim E, FHC Sá, YAO Duarte, RCB Oliveira, ML Lebrão. Prevalência e características das feridas em pessoas idosas residentes na comunidade. Rev Esc Enferm USP [Internet]. 2015 [citado em 24 mar 2020]; 49(Esp):51-7. Disponível em: http://www.scielo.br/pdf/reeusp/v49nspe/1980-220X-reeusp-49-spe-0051.pdf

2. Vieira CPB, Araújo TME. Prevalência e fatores associados a feridas crônicas em idosos na atenção básica. Rev Esc Enferm USP [Internet]. 2018 [citado em 24 mar 2020]; 52:e03415. DOI: http://dx.doi.org/10.1590/S1980-220X2017051303415

3. Kim CG. Avaliação e manejo de clientes com distúrbios vasculares e problemas na circulação periférica. In: Hinkle JL, Cheever KH. Brunner \& Suddarth: Tratado de Enfermagem Médicocirúrgica. 13o ed. Rio de Janeiro: Guanabara Koogan; 2018. Capítulo 30. p. 852

4. Almeida WA, Ferreira AM, Ivo ML, Rigotti MA, Barcelos LS, Silva ALNV. Fatores associados à qualidade de vida de pessoas com feridas complexas crônicas. Rev Pesqui. [Internet]. 2018 [citado em 22 mar 2019]; 10(1):9-16. DOI: 10.9789/2175-5361.2018.v10i1.9-16

5. Lentsck MH, Baratieri T, Trincaus MR, Mattei AP, Miyahara CTS. Qualidade de vida relacionada a aspectos clínicos em pessoas com ferida crônica. Rev Esc Enferm USP [Internet]. 2018 [citado em 24 mar 2020]; 52:e03384. DOI: http://dx.doi.org/10.1590/S1980220X2017004003384 
6. Instituto Brasileiro de Geografia e Estatística. Censo Demográfico 2010 [Internet]. Rio de Janeiro: IBGE; 2010 [citado em 13 jan 2019]. Disponível em: https://ww2.ibge.gov.br/home/estatistica/populacao/censo2010/default.shtm

7. Fleck MP, Chachamovich E, Trentini C. Development and validation of the Portuguese version of the WHOQOL-OLD module. Rev Saúde Pública [Internet] 2006 [citado em 20 jan 2019]; 40(5):785-91. DOI: http://dx.doi.org/10.1590/S0034-89102006000600007

8. Pedroso B, Pilatti LA, Gutierrez GL. Cálculo dos escores e estatística descritiva do WHOQOLOLD pelo Microsoft Excel. Geriatr Gerontol. [Internet]. 2010 [citado em 18 jan 2019]; 4(4):2149. Disponível

em:

http://fefnet178.fef.unicamp.br/ojs/index.php/fef/article/viewFile/622/391

9. Bardin L. Análise de conteúdo. São Paulo: Edições 70; 2011.

10. Vieira CPB, Furtado AS, Almeida PCD, Luz MHBA, Pereira AFM. Prevalência e caracterização de feridas crônicas em idosos assistidos na atenção básica. Rev Baiana Enferm [Internet]. 2017 [citado em 22 mar 2019]; 31(3):e17397. DOI: http://dx.doi.org/10.18471/rbe.v31i3.17397

11. Elias HC, Marzola TS, Molina NPFM, Assunção LM, Rodrigues LR, Tavares DMS. Relation between family functionality and the household arrangements of the elderly. Rev Bras Geriatr Gerontol. [Internet]. 2018 [citado em 22 mar 2019]; 21(5):562-9. DOI: http://dx.doi.org/10.1590/1981-22562018021.180081

12. Melo NCV, Teixeira KMD, Barbosa TL, Montoya AJA, Silveira MB. Arranjo domiciliar de idosos no Brasil: análises a partir da Pesquisa Nacional por Amostra de Domicílios (2009). Rev Bras Geriatr Gerontol. [Internet]. 2016 [citado em 29 mar 2020]; 19(1):139-51. DOI: https://doi.org/10.1590/1809-9823.2016.15011

13. Oliveira ALB, Menezes TMO. Significado da religião/religiosidade para a pessoa idosa. Rev Bras Enferm. [Internet]. 2018 [citado em 22 mar 2019]; 71(supl 2):823-9. DOI: http://dx.doi.org/10.1590/0034-7167-2017-0120

14. Barbosa KTB, Costa KNFM, Pontes MLF, Batista PSS, Oliveira FMLO, Fernandes MGM. Envelhecimento e vulnerabilidade individual: um panorama dos idosos vinculados à estratégia saúde da família. Texto Contexto Enferm [Internet]. 2017 [citado em 26 mar 2020]; 26(2):e2700015. DOI: https://doi.org/10.1590/0104-07072017002700015

15. Borges EL, Nascimento Filho HM, Pires Júnior JF. Prevalência de lesões crônicas de município da Zona da Mata Mineira (Brasil). Rev Min Enferm. [Internet]. 2018 [citado em 22 mar 2019]; 22:e1143. DOI: http://www.dx.doi.org/10.5935/1415-2762.20180074

16. Cortez ACL, Menezes JMMM, Brandão PP, Silva GCB, Dantas EHM. Correlação entre os testes de avaliação da capacidade funcional de idosos participantes de um projeto de inclusão social na cidade de Teresina - Piauí. J Health Sci. [Internet]. 2018 [citado em 22 mar 2019]; 20(4):27782. DOI: http://dx.doi.org/10.17921/2447-8938.2018v20n4p277-282

17. Martins JJ, Schneider DG, Coelho FL, Nascimento ERP, Albuquerque GL, Erdmann AL, et al. Avaliação da qualidade de vida de idosos que recebem cuidados domiciliares. Acta Paul Enferm. [Internet]. 2009 [citado em 10 fev 2019]; 22(3):265-71. DOI: http://dx.doi.org/10.1590/S0103-21002009000300005

18. Silveira PM, Borgatto AF, Silva KS, Oliveira ESA, Barros MVG, Nahas MV. Criação de uma escala de satisfação com a vida por meio da Teoria da Resposta ao Item. J Bras Psiquiatr. [Internet]. 2015 [citado em 22 mar 2019]; 64(4):272-8. DOI: http://dx.doi.org/10.1590/00472085000000089

19. Gomes E, Donoso MTV, Werli-Alvarenga A, Goveia VR. Compreendendo os significados de se conviver com ferida crônica. Rev Enferm Atenção Saúde [Internet]. 2018 [citado em 22 mar 2019]; 7(2):176-88. Disponível

em: http://seer.uftm.edu.br/revistaeletronica/index.php/enfer/article/view/2396/pdf 20. Ministério da Saúde (Brasil). Envelhecimento ativo: uma política de saúde [Internet]. Brasília, DF: Ministério da Saúde; 2005 [citado em 15 fev 2019]. Disponível em: http://bvsms.saude.gov.br/bvs/publicacoes/envelhecimento_ativo.pdf 
21. Leal TS, Oliveira BG, Bomfim ES, Figueredo NL, Souza AS, Santos ISC. Percepção de pessoas com a ferida crônica. Rev Enferm UFPE on line [Internet]. 2017 [citado em 22 mar 2019]; 11(3):1156-62. Disponível

em: https://periodicos.ufpe.br/revistas/revistaenfermagem/article/viewFile/13490/16210 22. Aguiar ACSA, Sadigursky D, Martins LA, Menezes TMO, Santos ALS, Reis LA. Repercussões sociais vivenciadas pela pessoa idosa com úlcera venosa. Rev Gaúcha Enferm [Internet]. 2016 [citado em 22 mar 2019]; 37(3):e55302. DOI: http://dx.doi.org/10.1590/19831447.2016.03.55302

23. Santos LSF, Camacho ACLF, Oliveira BGRB, Nogueira GA, Joaquim FL. Influência da úlcera venosa na qualidade de vida dos pacientes: revisão integrativa. Rev Enferm UFPE on line [Internet]. 2015 [citado em 22 mar 2019]; 9(supl 3):7710-22. DOI: 10.5205/reuol.7049-614521-ED.0903supl201526

\section{CONTRIBUIÇÕES}

Elayne Gonçalves Rodrigues do Nascimento contribuiu na concepção, coleta e análise de dados, redação e revisão. Giovanna Gabrielly Custódio Macêdo, Arthur Alexandrino, Karla Karolline Barreto Cardins e Fernanda Teixeira de Souza participaram na redação e revisão. Matheus Figueiredo Nogueira atuou na concepção, coleta e análise de dados e, revisão.

\section{Como citar este artigo (Vancouver)}

Nascimento EGR, Macêdo GGC, Alexandrino A, Cardins KKB, Souza FT, Nogueira MF. Percepção da qualidade de vida de idosos com ferida crônica. REFACS [Internet]. 2020 [citado em inserir dia, mês e ano de acesso]; 8(3):359-369. Disponível em: inserir link de acesso. DOI: inserir link do DOI.

\section{Como citar este artigo (ABNT)}

NASCIMENTO, E. G. R.; MACÊDO, G. G. C.; ALEXANDRINO, A.; CARDINS, K. K. B.; SOUZA, F. T.; NOGUEIRA, M. F. Percepção da qualidade de vida de idosos com ferida crônica. REFACS, Uberaba, MG, v. 8, n. 3, p. 359-369, 2020. Disponível em: inserir link de acesso. Acesso em: inserir dia, mês e ano de acesso. DOI: inserir link do DOI.

\section{Como citar este artigo (APA)}

Nascimento, E.G.R., Macêdo, G.G.C., Alexandrino, A., Cardins, K.K.B., Souza, F.T. \& Nogueira, M.F. (2020). Percepção da qualidade de vida de idosos com ferida crônica. REFACS, 8(3), 359369. Recuperado em: inserir dia, mês e ano de acesso de inserir link de acesso. DOI: inserir link do DOI 